\title{
An improved preparation of isatins from indoles
}

\author{
Jiro Tatsugi,* Tong Zhiwei, and Yasuji Izawa \\ Department of Applied Chemistry, Faculty of Engineering, Aichi Institute of Technology, \\ Yachigusa, Yakusa-cho, Toyota 470-0392, Japan \\ E-mail: jtatsugi@ac.aitech.ac.jp
}

(received 30 May 01; accepted 14 Oct 01; published on the web 22 Oct 01)

\begin{abstract}
A convenient method has been developed for the conversion of indoles into isatin derivatives in good to excellent yields. The general process utilizes our efficient one-pot method for bromination and oxidation with an N-bromosuccinimide - dimethyl sulfoxide reagent. 1-Alkyl-7azaindoles are readily available in excellent yields from the reaction of the sodium salt of 7azaindole with appropriate alkyl halides in dimethylacetamide. Similar reactions with 1-alkyl-5cyanoindoles and indole gave 1-alkyl-5-cyanoisatins and isatin, respectively.
\end{abstract}

Key words: Indoles, isatin derivatives, $N$-bromosuccinimide-dimethyl sulfoxide oxidation

\section{Introduction}

In connection with research developed in our studies on the photochemical behavior of cyclic vicinal polycarbonyl compounds, ${ }^{1-3}$ we needed to prepare isatins as structurally related compounds to investigate the photochemical reactions of heterocyclic vicinal polycarbonyls. Recently, much attention has been paid to isatins in organic synthesis. ${ }^{2-8}$ We reported one-pot synthesis of several vicinal polycarbonyl compounds via $\alpha$-bromo carbonyl derivatives from $\alpha$ methylene carbonyl compounds by N-bromosuccinimide (NBS) -DMSO oxidation. ${ }^{9}$ This oxidation method with NBS-DMSO reagent prompted us to explore an improved synthesis of isatins from indoles. We have examined the applicability of this NBS-DMSO oxidation to the conversion of 1-methyl-7-azaindole 2a into 1-methyl-7-azaisatin 3a as shown in Scheme 1. Initially, we prepared 1- methyl-7-azaindole 2a from the reaction of the sodium salt of 7azaindole 1 with methyl iodide in dimethylacetamide. It was found that the oxidation of $2 \mathrm{a}$ with NBS in DMSO to $3 \mathrm{a}$ was carried out at $60^{\circ} \mathrm{C}$ for $6 \mathrm{~h}$ under ambient pressure and then at above $80^{\circ} \mathrm{C}$ for $20 \mathrm{~h}$ under reduced pressure to remove the generated hydrogen bromide. In this paper, we describe this improved method for the preparation of the isatin derivatives $\mathbf{3}$ from indoles $\mathbf{1}$. 
<smiles>c1cnc2[nH]ccc2c1</smiles>

1

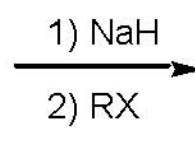

DMAC $\mathrm{X}=\mathrm{Br}, \mathrm{I}$<smiles>[R]n1ccc2cccnc21</smiles>

2a,b,c$$
\text { (n) }
$$

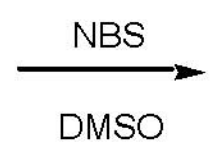

DMSO<smiles>[R]N1C(=O)C(=O)c2cccnc21</smiles>

$3 a, b, c$

a: $R=M e, b: R=E t, c: R=B n$

\section{Scheme 1}

\section{Results and Discussion}

Alkyl-7-azaindoles 2a, 2b, and 2c were prepared in excellent yields by the reaction of the sodium salt of 1 with appropriate alkyl halides in dimethylacetamide at room temperature. The resulting 1-alkylated derivatives 2 were subsequently oxidized to 3 with the NBS-DMSO reagent. When oxidation of 2a with NBS in anhydrous DMSO was carried out at room temperature for $12 \mathrm{~h}$, a small amount of 3a was detected by GC-MS analysis. Unfortunately, when this oxidation reaction was performed at above 80 under ambient pressure, rapid decomposition of DMSO by the generated hydrogen bromide predominantly proceeded. ${ }^{10,11}$ Therefore, it was necessary in the present oxidation reaction to prevent the acid-catalyzed decomposition of DMSO. In order to remove the generated hydrogen bromide, the oxidation with NBS in DMSO at above $80^{\circ} \mathrm{C}$ was carried out under reduced pressure. Thus, the desired 3a was obtained in 95\% yield by treatment of 2a with NBS in DMSO at $60^{\circ} \mathrm{C}$ for $6 \mathrm{~h}$ under ambient pressure and then at above $80^{\circ} \mathrm{C}$ for $20 \mathrm{~h}$ under reduced pressure to remove the generated hydrogen bromide. Similarly, 1-ethyl- and 1-benzyl-7-azaindoles $2 \mathbf{b}$ and 2c were converted to the corresponding 1-alkylated 7-azaisatins $3 \mathbf{b}$ and 3c in 95 and 92\% yields, respectively.

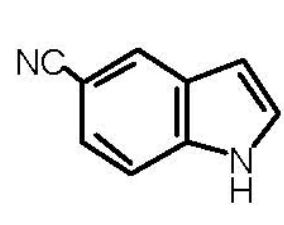

1d

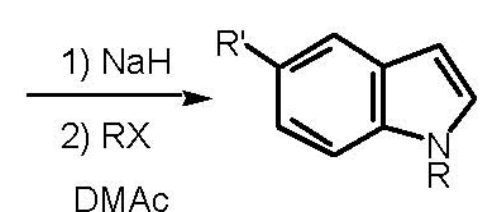

$2 e, f, g, h$

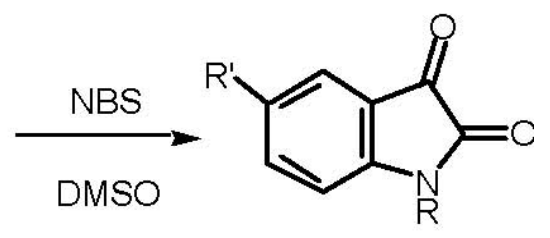

$3 e, f, g, h$

$$
\begin{array}{lc}
\quad \text { R } & R^{\prime} \\
\text { e }=\mathrm{Me} & \mathrm{CN} \\
\mathrm{f}=\mathrm{Et} & \mathrm{CN} \\
\mathrm{g}=\mathrm{Bn} & \mathrm{CN} \\
\mathrm{h}=\mathrm{H} & \mathrm{H}
\end{array}
$$

Scheme 2

In a similar manner, 5-cyanoindole 1d gave the corresponding 1-alkyled 5-cyanoisatins 3e, 3f, 
and $\mathbf{3 g}$ in fair yields via 1-alkyl-5-cyanoindoles $\mathbf{2 e}$, $\mathbf{2 f}$, and $\mathbf{2 g}$, and indole $\mathbf{1 h}$ gave isatin $\mathbf{3 h}$ in $90 \%$ yield (Scheme 2). A plausible reaction pathway for the formation of 3 is illustrated in Scheme 3. The initial bromination of $\mathbf{2}$ would yield 2,3-dibromo derivative A. The dibromoindole is brominated a third time at $\mathbf{C}-3$, generating a 2,3,3-tribromo-3H-indolium salt $\mathbf{B}$ which is hydrolysed during work up with water to give the isatin 3 .

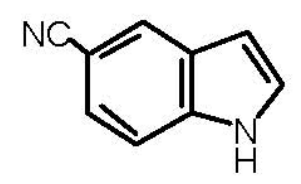

1d

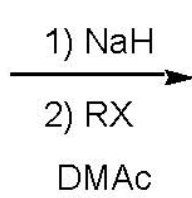

$\mathrm{X}=\mathrm{Br}, \mathrm{I}$<smiles>[R]c1ccc2c(ccn2[R])c1</smiles>

$2 e, f, g, h$

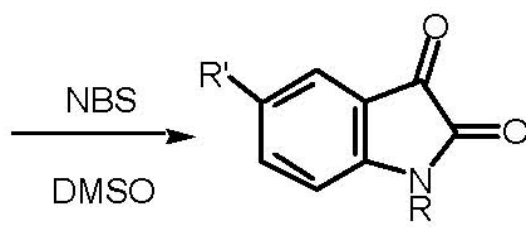

$3 e, f, g, h$

$$
\begin{array}{lc}
\quad \text { R } & R^{\prime} \\
\mathrm{e}=\mathrm{Me} & \mathrm{CN} \\
\mathrm{f}=\mathrm{Et} & \mathrm{CN} \\
\mathrm{g}=\mathrm{Bn} & \mathrm{CN} \\
\mathrm{h}=\mathrm{H} & \mathrm{H}
\end{array}
$$

\section{Scheme 3}

To confirm the reaction pathway to 3 , the reaction of 2,3-dibromo-1-methyl-7-azaindole which was prepared by the bromination of $\mathbf{2 a}$ with two equivalents of bromine in dichloromethane with DMSO was carried out under the above similar oxidation conditions to afford $\mathbf{3 a}$ in excellent yield. The results indicate that the formation of $\mathbf{3}$ is considered to proceed via A.

In summary, a convenient synthesis of isatin derivatives from commercially available indoles via 1 -alkylindoles is described.

\section{Experimental Section}

General Procedures. Melting points were determined on a Yanagimoto hot-stage apparatus and are uncorrected. IR spectra were recorded on a Nihon Bunko 7300 FT-IR spectrometer in $\mathrm{KBr}$ with absorptions in $\mathrm{cm}^{-1}$. The UV-vis spectra were recorded using a Shimadzu UV-3100S spectrophotometer. ${ }^{1} \mathrm{H}$ and ${ }^{13} \mathrm{C}$ NMR spectra were recorded on a Varian Gemini-200 from a solution $\mathrm{CDCl}_{3}$ of the product. ${ }^{1} \mathrm{H}$ chemical shifts are expressed as $\delta$ values (ppm) relative to TMS as an internal standard. MS spectra and HRMS were recorded on Hitachi 80-B spectrometer. Elemental analyses were performed at the Center of Instrumental Analysis, Meijo University, Nagoya, Japan. For column chromatography, silica gel (nacalai tesque, $230-400$ mesh) was used. Commercal dimethyl sulfoxide was purified by drying over calcium hydride and distillation. 7-Azaindole (Aldrich), indole, methyl iodide, ethyl iodide, benzyl bromide, and NBS were commercially available and were used without purification.

\section{General procedure I. Alkylation of 7-azaindole}


Sodium hydride ( $0.1 \mathrm{~g}, 4 \mathrm{mmol})$ free of mineral oil was added to 7-azaindole $\mathbf{1}(0.35 \mathrm{~g}, 3 \mathrm{mmol})$ in dimethylacetamide $(10 \mathrm{~mL})$ under an inert atmosphere. After $30 \mathrm{~min}$, the appropriate alkyl halide $(3.5 \mathrm{mmol})$ was added slowly as a solution in dimethylacetamide $(2 \mathrm{~mL})$, and the solution was stirred at $\mathrm{rt}$ for $12 \mathrm{~h}$ to give a pale yellow solution. The reaction was quenched with water $(20 \mathrm{~mL})$ and extracted with dichloromethane. The combined extracts were washed three times with distilled water. After drying $\left(\mathrm{MgSO}_{4}\right)$ the dichloromethane layer and removal of the solvent, the residue was purified by chromatography on silica gel with dichloromethane as an eluent to give the respective 1-alkyl-7-azaindoles.

1-Methyl-7-azaindole (2a). According to procedure I, 7-azaindole was converted to 2a; yield: $0.38 \mathrm{~g}$ (95\%); pale yellow oil. IR (neat) $\mathrm{n}=1596,1571,1516,1440,1410,1348,1315,1279$, 797, 773, $719 \mathrm{~cm}^{-1} ;{ }^{1} \mathrm{H}$ NMR $\left(\mathrm{CDCl}_{3}\right) \delta: 3.86\left(\mathrm{~s}, 3 \mathrm{H}, \mathrm{CH}_{3}\right), 6.42(\mathrm{~d}, J=3.6 \mathrm{~Hz}, 1 \mathrm{H}, 3-\mathrm{H}), 7.02$ (dd, $J=4.8,7.8 \mathrm{~Hz}, 1 \mathrm{H}, 5-\mathrm{H}), 7.13$ (d, J=3.6 Hz, 1H, 2-H), 7.88 (dd, $J=1.6,7.8 \mathrm{~Hz}, 1 \mathrm{H}, 4-\mathrm{H})$, $8.33(\mathrm{dd}, J=1.6,4.8 \mathrm{~Hz}, 1 \mathrm{H}, 6-\mathrm{H}) ;{ }^{13} \mathrm{C} \mathrm{NMR}\left(\mathrm{CDCl}_{3}\right) \delta: 31.1,99.2,115.3,120.4,128.6,128.9$, 142.5, 147.5; MS m/z (\%) = $132\left(\mathrm{M}^{+}, 93\right), 131(100), 103(63), 65$ (57). Anal. Calcd for $\mathrm{C}_{8} \mathrm{H}_{8} \mathrm{~N}_{2}$ : C, 72.70; H, 6.10; N, 21.20. Found: C, 72.25; H, 6.22; N, 20.97.

1-Ethyl-7-azaindole (2b). Yield 92\%, pale yellow oil; IR (neat) $\mathrm{n}=1594,1569,1508,1428$, $1403,1358,1347,1319,1305,1268,1206,797 \mathrm{~cm}^{-1} ;{ }^{1} \mathrm{H} \mathrm{NMR}\left(\mathrm{CDCl}_{3}\right) \delta: 1.45$ (t, $J=7.2 \mathrm{~Hz}$, $\left.3 \mathrm{H}, \mathrm{CH}_{3}\right), 4.32\left(\mathrm{q}, J=7.2 \mathrm{~Hz}, 2 \mathrm{H}, \mathrm{CH}_{2}\right), 6.42(\mathrm{~d}, J=3.6 \mathrm{~Hz}, 1 \mathrm{H}, 3-\mathrm{H}), 7.02(\mathrm{dd}, J=4.8,7.8 \mathrm{~Hz}$, $1 \mathrm{H}, 5-\mathrm{H}$ ), 7.19 (d, $J=3.6 \mathrm{~Hz}, 1 \mathrm{H}, 2-\mathrm{H}), 7.87$ (dd, $J=1.6,7.8 \mathrm{~Hz}, 1 \mathrm{H}, 4-\mathrm{H}), 8.32$ (dd, $J=1.6,4.8$ $\mathrm{Hz}, 1 \mathrm{H}, 6-\mathrm{H}) ;{ }^{13} \mathrm{C} \mathrm{NMR}\left(\mathrm{CDCl}_{3}\right) \delta: 15.5,39.1,99.2,115.3,120.6,127.1,128.6,142.4,1467.0$; MS m/z (\%) = $146\left(\mathrm{M}^{+}, 58\right), 131(41), 118(100), 91(10), 65(11)$. Anal. Calcd for $\mathrm{C}_{9} \mathrm{H}_{10} \mathrm{~N}_{2}: \mathrm{C}$, 73.94; H, 6.89; N, 19.16. Found: C, 73.47; H, 7.01; N, 18.89.

Benzyl-7-azaindole (2c). Yield 96\%, pale yellow oil; IR (neat) $\mathrm{n}=1592,1568,1511,1494$, $1454,1435,1421,1349,1314,1211,800,749,733 \mathrm{~cm}^{-1} ;{ }^{1} \mathrm{H} \mathrm{NMR}\left(\mathrm{CDCl}_{3}\right) \delta: 5.50\left(\mathrm{~s}, 2 \mathrm{H}, \mathrm{CH}_{2}\right)$, 6.47 (d, $J=3.6 \mathrm{~Hz}, 1 \mathrm{H}, 3-\mathrm{H}), 7.07$ (dd, $J=4.8,7.8 \mathrm{~Hz}, 1 \mathrm{H}, 5-\mathrm{H}), 7.16(\mathrm{~d}, J=3.6 \mathrm{~Hz}, 1 \mathrm{H}, 2-\mathrm{H})$, $7.2-7.3\left(\mathrm{~m}, 5 \mathrm{H}, \mathrm{C}_{6} \mathrm{H}_{5}\right), 7.92$ (dd, $\left.J=1.6,7.8 \mathrm{~Hz}, 1 \mathrm{H}, 4-\mathrm{H}\right), 8.34$ (dd, $\left.J=1.6,4.8 \mathrm{~Hz}, 1 \mathrm{H}, 6-\mathrm{H}\right)$; ${ }^{13} \mathrm{C}$ NMR $\left(\mathrm{CDCl}_{3}\right) \delta: 47.9,100.2,115.8,120.6,127.5,127.6,128.0,128.7,129.0,137.7,142.7$, 147.4; MS m/z (\%) = $208\left(\mathrm{M}^{+}, 95\right), 207$ (100), 131 (43), 103 (12), 91 (95), 66 (32). Anal. Calcd for $\mathrm{C}_{14} \mathrm{H}_{12} \mathrm{~N}_{2}$ : C, 80.74; H, 5.81; N, 13.45. Found: C, 80.31; H, 5.89; N, 13.36.

1-Methyl-5-cyanoindole (2e). According to procedure I, 5-cyanoindole (1d) was converted to 2e; yield: (91\%); pale yellow oil. IR (neat) $\mathrm{n}=2221(\mathrm{CN}), 1611,1513,1488,1342,1292$, $1249 \mathrm{~cm}^{-1} ;{ }^{1} \mathrm{H}$ NMR $\left(\mathrm{CDCl}_{3}\right) \delta: 3.82\left(\mathrm{~s}, 3 \mathrm{H}, \mathrm{CH}_{3}\right), 6.55(\mathrm{~d}, J=3.0 \mathrm{~Hz}, 1 \mathrm{H}, 3-\mathrm{H}), 7.16(\mathrm{~d}, J=3.0$ $\mathrm{Hz}, 1 \mathrm{H}, 2-\mathrm{H}), 7.34$ (d, $J=8.4 \mathrm{~Hz}, 1 \mathrm{H}, 6-\mathrm{H}), 7.43$ (d, $J=8.4 \mathrm{~Hz}, 1 \mathrm{H}, 7-\mathrm{H}), 7.94(\mathrm{~s}, 1 \mathrm{H}, 4-\mathrm{H}) ;{ }^{13} \mathrm{C}$ $\operatorname{NMR}\left(\mathrm{CDCl}_{3}\right) \delta: 33.0,102.2,102.5,110.0,120.8,124.4,126.4,128.2,131.1,138.2 ; \mathrm{MS} \mathrm{m} / \mathrm{z}$ $(\%)=156\left(\mathrm{M}^{+}, 100\right), 141(28), 128(30), 113(55), 101(32)$.

Ethyl-5-cyanoindole (2f). Yield 90\%, pale yellow oil; IR (neat) $\mathrm{n}=2220(\mathrm{CN}), 1609,1452$, $1401,1340,1294,1223 \mathrm{~cm}^{-1} ;{ }^{1} \mathrm{H}$ NMR $\left(\mathrm{CDCl}_{3}\right) \delta: 1.47$ (t, $\left.J=7.4 \mathrm{~Hz}, 3 \mathrm{H}, \mathrm{CH}_{3}\right), 4.20$ (q, $J=7.4$ $\left.\mathrm{Hz}, 2 \mathrm{H}, \mathrm{CH}_{2}\right), 6.57(\mathrm{~d}, J=3.4 \mathrm{~Hz}, 1 \mathrm{H}, 3-\mathrm{H}), 7.24(\mathrm{~d}, J=3.4 \mathrm{~Hz}, 1 \mathrm{H}, 2-\mathrm{H}), 7.37$ (d, $J=8.4 \mathrm{~Hz}$, $1 \mathrm{H}, 6-\mathrm{H}), 7.42(\mathrm{~d}, J=8.4 \mathrm{~Hz}, 1 \mathrm{H}, 7-\mathrm{H}), 7.95(\mathrm{~s}, 1 \mathrm{H}, 4-\mathrm{H}) ;{ }^{13} \mathrm{C} \mathrm{NMR}\left(\mathrm{CDCl}_{3}\right) \delta: 15.3,41.2$, 102.2, 102.5, 110.0, 120.9, 124.2, 126.5, 128.2, 129.3, 137.1; MS m/z $(\%)=170\left(\mathrm{M}^{+}, 90\right), 155$ 
(100), 142 (25), $128(20), 115$ (35).

Benzyl-5-cyanoindole (2g). Yield 85\%, colorless plates; mp $108-109{ }^{\circ} \mathrm{C}\left(\mathrm{CH}_{2} \mathrm{Cl}_{2}\right)$; IR $(\mathrm{KBr}) \mathrm{n}$ $=2223(\mathrm{CN}), 1606,1483,1452,1483,1341,768,740,725 \mathrm{~cm}^{-1} ;{ }^{1} \mathrm{H}$ NMR $\left(\mathrm{CDCl}_{3}\right) \delta: 5.32(\mathrm{~s}$, $\left.2 \mathrm{H}, \mathrm{CH}_{2}\right), 6.61(\mathrm{~d}, J=3.2 \mathrm{~Hz}, 1 \mathrm{H}, 3-\mathrm{H}), 7.05-7.10(\mathrm{~m}, 2 \mathrm{H}, 6-\mathrm{H}$ and $7-\mathrm{H}), 7.24(\mathrm{~d}, J=3.2 \mathrm{~Hz}$, $1 \mathrm{H}, 2-\mathrm{H}), 7.28-7.38\left(\mathrm{~m}, 5 \mathrm{H}, \mathrm{C}_{6} \mathrm{H}_{5}\right), 7.95(\mathrm{~s}, 1 \mathrm{H}, 4-\mathrm{H}) ;{ }^{13} \mathrm{C} \mathrm{NMR}\left(\mathrm{CDCl}_{3}\right) \delta: 50.3,102.5,102.7$, $110.5,120.7,124.5,126.5,126.6,128.0,128.3,128.9,130.6,137.7 ; \mathrm{MS} \mathrm{m} / \mathrm{z}(\%)=232\left(\mathrm{M}^{+}\right.$, 85), $141(25), 114(27), 91$ (100).

General procedure II. Synthesis of 1-alkylisatins by oxidation of 1-alkylindoles with NBSDMSO

A mixture of 1-alkyl-7-azaindole (2.4 mmol), NBS (0.90 g, $5.0 \mathrm{mmol})$ and anhydrous DMSO $(20 \mathrm{~mL})$ was stirred at $60{ }^{\circ} \mathrm{C}$ for $6 \mathrm{~h}$ and then above $80{ }^{\circ} \mathrm{C}$ for $20 \mathrm{~h}$ under reduced pressure. The progress of the reaction was monitored by GC and GC-MS. After disappearance of 2, the reaction mixture was poured into water $(50 \mathrm{~mL})$, followed by extracting with dichloromethane $(10 \mathrm{~mL} \times 3)$. The combined extracts were washed three times with distilled water and dried $\left(\mathrm{MgSO}_{4}\right)$. After removal of the solvent, the residue was purified chromatography on silica gel with dichloromethane as an eluent to give the pure product 3a-c and 3e-g.

1-Methyl-7-azaisatin (3a). Yield $=95 \%$, yellow plates; mp $160-161{ }^{\circ} \mathrm{C}\left(\mathrm{CH}_{2} \mathrm{Cl}_{2}\right) ; \mathrm{IR}(\mathrm{KBr}) \mathrm{n}=$ $1750(\mathrm{C}=\mathrm{O}), 1607,1594,1458 \mathrm{~cm}^{-1} ; \mathrm{UV}\left(\mathrm{CH}_{2} \mathrm{Cl}_{2}\right): \lambda_{\max }(\log \varepsilon)=275 \mathrm{~nm}$ (3.396), 406 (2.672); ${ }^{1} \mathrm{H}$ NMR $\left(\mathrm{CDCl}_{3}\right) \delta: 3.36\left(\mathrm{~s}, 3 \mathrm{H}, \mathrm{CH}_{3}\right), 7.10(\mathrm{dd}, 1 \mathrm{H}, J=7.2,7.5 \mathrm{~Hz}, 5-\mathrm{H}), 7.84(\mathrm{~d}, 1 \mathrm{H}, J=7.5$ $\mathrm{Hz}, 4-\mathrm{H}), 8.47$ (d, $1 \mathrm{H}, J=7.2 \mathrm{~Hz}, 6-\mathrm{H}) ;{ }^{13} \mathrm{C} \mathrm{NMR} \delta: 25.0,112.0,119.6,132.8,155.8,158.3$, 163.8, 181.9; MS m/z (\%) = $162\left(\mathrm{M}^{+}, 58\right), 134$ (34), 105 (40), 75 (100); HRMS Calcd for $\mathrm{C}_{8} \mathrm{H}_{6} \mathrm{~N}_{2} \mathrm{O}_{2}$ 162.0428, Found 162.0429; Anal. Calcd for $\mathrm{C}_{8} \mathrm{H}_{6} \mathrm{~N}_{2} \mathrm{O}_{2}$ : C, 59.26; H, 3.73; N, 17.28. Found: C, 59.79; H, 3.86; N, 17.00 .

1-Ethyl-7-azaisatin (3b). Yield $=95 \%$, yellow plates; mp $127-128{ }^{\circ} \mathrm{C}\left(\mathrm{CH}_{2} \mathrm{Cl}_{2}\right)$; $\mathrm{IR}(\mathrm{KBr}) \mathrm{n}=$ $1742(\mathrm{C}=\mathrm{O}), 1607,1593,1358 \mathrm{~cm}^{-1} ; \mathrm{UV}\left(\mathrm{CH}_{2} \mathrm{Cl}_{2}\right): \lambda_{\max }(\log \varepsilon)=275 \mathrm{~nm}(2.991), 409$ (2.230); ${ }^{1} \mathrm{H}$ NMR $\left(\mathrm{CDCl}_{3}\right) \delta: 1.36(\mathrm{t}, \mathrm{J}=7.2 \mathrm{~Hz}, 3 \mathrm{H}),, 3.93\left(\mathrm{q}, 2 \mathrm{H}, \mathrm{J}=7.2 \mathrm{~Hz}, \mathrm{CH}_{2}\right), 7.11(\mathrm{dd}, 1 \mathrm{H}, \mathrm{J}=7.2$, $7.5 \mathrm{~Hz}), 7.85(\mathrm{~d}, 1 \mathrm{H}, \mathrm{J}=7.5 \mathrm{~Hz}), 8.47(\mathrm{~d}, 1 \mathrm{H}, \mathrm{J}=7.2 \mathrm{~Hz}) ;{ }^{13} \mathrm{C} \mathrm{NMR} \delta: 12.8,34.1,112.0,119.4$, 132.8, 155.6, 157.9, 163.6, 182.1; MS m/z (\%) 176 (M+, 74), 147 (10), 133 (46), 120 (100); HRMS Calcd for $\mathrm{C}_{9} \mathrm{H}_{8} \mathrm{~N}_{2} \mathrm{O}_{2}$ 176.0585, Found 176.0561. Anal. Calcd for $\mathrm{C}_{9} \mathrm{H}_{8} \mathrm{~N}_{2} \mathrm{O}_{2}$ : C, 61.36; H, 4.58; N, 15.90. Found: C, 61.58; H, 4.50; N, 15.91.

1-Benzyl-7-azaisatin (3c). Yield $=92 \%$, yellow plates; mp $187-188^{\circ} \mathrm{C}\left(\mathrm{CH}_{2} \mathrm{Cl}_{2}\right), \mathrm{IR}(\mathrm{KBr}) \mathrm{n}=$ $1742(\mathrm{C}=\mathrm{O}), 1603,1592,1443 \mathrm{~cm}^{-1} ; \mathrm{UV}\left(\mathrm{CH}_{2} \mathrm{Cl}_{2}\right): \lambda_{\max }(\log \varepsilon)=277 \mathrm{~nm}(3.762), 423$ (2.626); ${ }^{1} \mathrm{H}$ NMR $\left(\mathrm{CDCl}_{3}\right) \delta: 5.03\left(\mathrm{~s}, 2 \mathrm{H}, \mathrm{CH}_{2}\right), 7.08(\mathrm{dd}, J=7.2,7.5 \mathrm{~Hz}, 1 \mathrm{H}, 5-\mathrm{H}), 7.26-7.52(\mathrm{~m}, 5 \mathrm{H}$, $\left.\mathrm{C}_{6} \mathrm{H}_{5}\right), 7.82(\mathrm{~d}, J=7.5 \mathrm{~Hz}, 1 \mathrm{H}, 4-\mathrm{H}), 8.46(\mathrm{~d}, J=7.2 \mathrm{~Hz}, 1 \mathrm{H}, 6-\mathrm{H}) ;{ }^{13} \mathrm{C} \mathrm{NMR} \delta: 42.7,112.1$, $119.6,128.1,128.7,128.8,132.9,135.4,155.7,158.1,163.5,181.8 ; \mathrm{MS} \mathrm{m} / \mathrm{z}(\%) 238\left(\mathrm{M}^{+}, 20\right)$, 210 (16), 181 (49), 147 (78), 119 (22), 92 (100); HRMS Calcd for $\mathrm{C}_{14} \mathrm{H}_{10} \mathrm{~N}_{2} \mathrm{O}_{2} 238.0741$, Found 238.0736. Anal. Calcd for $\mathrm{C}_{14} \mathrm{H}_{10} \mathrm{~N}_{2} \mathrm{O}_{2}$ : C, 70.58; H, 4.23; N, 11.76. Found: C, 70.30; H, 4.28; $\mathrm{N}, 11.72$. 
5-Cyano-1-methylisatin (3e). Yield $=26 \%$, orange needles; mp $222-223{ }^{\circ} \mathrm{C}\left(\mathrm{CH}_{2} \mathrm{Cl}_{2}\right), \mathrm{IR}(\mathrm{KBr})$ $\mathrm{n}=2232(\mathrm{CN}), 1743(\mathrm{C}=\mathrm{O}), 1621,1590,1489 \mathrm{~cm}^{-1} ; \mathrm{UV}\left(\mathrm{CH}_{2} \mathrm{Cl}_{2}\right): \lambda_{\max }(\log \varepsilon)=250 \mathrm{~nm}(4.637)$, 4 (2.672); ${ }^{1} \mathrm{H}$ NMR $\left(\mathrm{CDCl}_{3}\right) \delta: 3.33\left(\mathrm{~s}, 3 \mathrm{H}, \mathrm{CH}_{3}\right), 7.05(\mathrm{~d}, J=8.2 \mathrm{~Hz}, 1 \mathrm{H}, 7-\mathrm{H}), 7.88$ (s, 1H, 4$\mathrm{H}), 7.92(\mathrm{~d}, J=8.2 \mathrm{~Hz}, 1 \mathrm{H}, 6-\mathrm{H}) ;{ }^{13} \mathrm{C}$ NMR $\delta: 26.6,107.6,110.8,117.5,128.1,128.6,141.9$, 153.9, 157.4, 181.1; MS m/z (\%) $186\left(\mathrm{M}^{+}, 81\right), 158$ (40), 129 (100), 103 (60); HRMS Calcd for $\mathrm{C}_{10} \mathrm{H}_{6} \mathrm{~N}_{2} \mathrm{O}_{2}$ 186.0428, Found 186.0428 .

5-Cyano-1-ethylisatin (3f). Yield $=57 \%$, orange plates; mp $182-184{ }^{\circ} \mathrm{C}\left(\mathrm{CH}_{2} \mathrm{Cl}_{2}\right), \mathrm{IR}(\mathrm{KBr}) \mathrm{n}=$ $2227(\mathrm{CN}), 1745(\mathrm{C}=\mathrm{O}), 1616,1587,1489 \mathrm{~cm}^{-1} ; \mathrm{UV}\left(\mathrm{CH}_{2} \mathrm{Cl}_{2}\right): \lambda_{\max }(\log \varepsilon)=253 \mathrm{~nm}(4.417)$, 428 (2.681); ${ }^{1} \mathrm{H}$ NMR $\left(\mathrm{CDCl}_{3}\right) \delta: 1.34\left(\mathrm{t}, J=7.4 \mathrm{~Hz}, 3 \mathrm{H}, \mathrm{CH}_{3}\right), 3.85\left(\mathrm{q}, J=7.4 \mathrm{~Hz}, 2 \mathrm{H}, \mathrm{CH}_{2}\right)$, $7.08(\mathrm{~d}, J=8.2 \mathrm{~Hz}, 1 \mathrm{H}, 7-\mathrm{H}), 7.87(\mathrm{~s}, 1 \mathrm{H}, 4-\mathrm{H}), 7.90(\mathrm{~d}, J=8.2 \mathrm{~Hz}, 1 \mathrm{H}, 6-\mathrm{H}) ;{ }^{13} \mathrm{C} \mathrm{NMR} \delta$ : $12.4,35.4,107.3,110.9,117.4,117.6,128.8,141.9,153.3,157.1,181.5 ; \mathrm{MS} \mathrm{m} / \mathrm{z}(\%) 200$ (M , 82), 171 (8), 158 (26), 144 (100), 120 (83), 115 (34); HRMS Calcd for $\mathrm{C}_{11} \mathrm{H}_{8} \mathrm{~N}_{2} \mathrm{O}_{2} 200.0584$, Found 200.0557.

5-Cyano-1-benzylisatin (3g). Yield $=20 \%$, orange needles; mp $193-195^{\circ} \mathrm{C}\left(\mathrm{CH}_{2} \mathrm{Cl}_{2}\right), \mathrm{IR}(\mathrm{KBr})$ $\mathrm{n}=2227(\mathrm{CN}), 1742(\mathrm{C}=\mathrm{O}), 1619,1589,1483 \mathrm{~cm}^{-1} ; \mathrm{UV}\left(\mathrm{CH}_{2} \mathrm{Cl}_{2}\right): \lambda_{\max }(\log \varepsilon)=256 \mathrm{~nm}$ (4.242), 406 (2.591); ${ }^{1} \mathrm{H}$ NMR $\left(\mathrm{CDCl}_{3}\right) \delta: 4.98\left(\mathrm{~s}, 2 \mathrm{H}, \mathrm{CH}_{2}\right), 6.92(\mathrm{~d}, J=8.4 \mathrm{~Hz}, 1 \mathrm{H}, 7-\mathrm{H}), 7.32$ - $7.38\left(\mathrm{~m}, 5 \mathrm{H}, \mathrm{C}_{6} \mathrm{H}_{5}\right), 7.77(\mathrm{dd}, J=1.8,8.4 \mathrm{~Hz}, 1 \mathrm{H}, 6-\mathrm{H}), 7.87(\mathrm{~d}, J=1.8 \mathrm{~Hz}, 1 \mathrm{H}, 4-\mathrm{H}) ;{ }^{13} \mathrm{C}$ NMR $\delta: 44.4,107.7,111.9,117.3,117.6,127.3,128.6,128.8,129.3,133.3,141.7,153.2,157.5$, 181.1; MS m/z (\%) = 262 (M , 39), 205 (9), 171 (60), 114 (5), 91 (100); HRMS Calcd for $\mathrm{C}_{16} \mathrm{H}_{1} \mathrm{~N}_{2} \mathrm{O}_{2} 262.0741$, Found 262.0766.

Isatin $(3 \mathrm{~h}){ }^{12}$. Yield=94\%, orange crystal.

2,3-Dibromo-1-methyl-7-azaindole (A): Reaction of 1-methyl-7-azaindole $(0.2 \mathrm{~g}, 1.5 \mathrm{mmol})$ with bromine $(0.48 \mathrm{~g}, 3.0 \mathrm{mmol})$ in dichloromethane $(10 \mathrm{~mL})$ at $\mathrm{rt}$. for $12 \mathrm{~h}$ gave $\mathbf{A}$ in $80 \%$ yield, mp $90-92{ }^{\circ} \mathrm{C}\left(\mathrm{CH}_{2} \mathrm{Cl}_{2}\right), \mathrm{IR}(\mathrm{KBr}) \mathrm{n}=1566,1497,1482,1403,1318,1296,947,791,766$, $552 \mathrm{~cm}^{-1} ;{ }^{1} \mathrm{H}$ NMR $\left(\mathrm{CDCl}_{3}\right) \delta: 3.36\left(\mathrm{~s}, 3 \mathrm{H}, \mathrm{CH}_{3}\right), 7.11(\mathrm{dd}, J=5.2,7.8 \mathrm{~Hz}, 1 \mathrm{H}, 5-\mathrm{H}), 7.81(\mathrm{dd}, J$ $=1.6,7.8 \mathrm{~Hz}, 1 \mathrm{H}, 4-\mathrm{H}), 8.26(\mathrm{dd}, J=1.6,5.2 \mathrm{~Hz}, 1 \mathrm{H}, 6-\mathrm{H}) ;{ }^{13} \mathrm{C} \mathrm{NMR} \delta: 26.3,116.5,119.7$, 126.0, 128.1, 133.2, 150.1, 152.5; MS m/z $(\%)=292\left(\mathrm{M}^{+}+2,70\right), 290\left(\mathrm{M}^{+}, 100\right), 288\left(\mathrm{M}^{+}-2\right.$, 68), 211 (38), 209 (40), 130(50).

\section{References}

1. Tatsugi, J.; Ikuma, K. ; Izawa,Y. Tetrahedron Lett. 1995, 36, 8611.

2. Tatsugi, J.; Ikuma, K. ; Izawa,Y. Heterocycles 1996, 43, 7.

3. Tatsugi, J.; Hara,T.; Izawa,Y. Chem. Lett, 1997, 177.

4. Xue, J.; Zhang, Y.; Wang, X-I.; Fun, H. K.; Xu, J-H. Org. Lett. 2000, 2, 2583.

5. Nair, V.; Sheela, K. C.; Rath, N. P. Chem. Lett. 2000, 980.

6. Klumpp, D. A.; Yeung, K. Y.; Prakash, G. K. S.; Olah, G. A. J. Org. Chem. 1998, 63, 4481.

7. Hewawasam, P.; Erway, M. Tetrahedron Lett. 1998, 39, 3981.

8. Garden, S. J.; Torres,J. C.; Ferreira, A. A.; Silva, R. B.; Pinto, A. C. Tetrahedron Lett. 1997, 
38, 1501 .

9. Tatsugi, J. ; Izawa,Y. Synth. Commun. 1998, 28, 859.

10. Traynelis, V. J.; Hergenvother, W. C. J. Org. Chem. 1964, 29, 221.

11. Traynelis, V. J.; Hergenvother, W. C. J. Am. Chem. Soc. 1964, 86, 298.

12. Gassman, P. G., Cue, Jr., B. W.; Luh, T-Y., J. Org. Chem. 1977, 42, 1344. 